\title{
MicroRNA transcriptomic analysis of the sixth leaf of maize (Zea mays L.) revealed a regulatory mechanism of jointing stage heterosis
}

Gege Hou, Yahui Dong, Fangfang Zhu, Qiannan Zhao, Tianyi Li, Dandan Dou, Xingli Ma, Liancheng Wu, Lixia Ku and Yanhui Chen ${ }^{*}$

\begin{abstract}
Background: Zhengdan 958 (Zheng $58 \times$ Chang 7-2), a commercial hybrid that is produced in a large area in China, is the result of the successful use of the heterotic pattern of Reid $\times$ Tang-SPT. The jointing stage of maize is the key period from vegetative to reproductive growth, which determines development at later stages and heterosis to a certain degree. MicroRNAs (miRNAs) play vital roles in the regulation of plant development, but how they function in the sixth leaf at the six-leaf (V6) stage to influence jointing stage heterosis is still unclear.

Result: Our objective was to study miRNAs in four hybrid combinations developed in accordance with the Reid $x$ Tang-SPT pattern, Zhengdan 958, Anyu 5 (Ye $478 \times$ Chang 7-2), Ye $478 \times$ Huangzaosi, Zheng $58 \times$ Huangzaosi, and their parental inbred lines to explore the mechanism related to heterosis. A total of 234 miRNAs were identified in the sixth leaf at the V6 stage, and 85 miRNAs were differentially expressed between the hybrid combinations and their parental inbred lines. Most of the differentially expressed miRNAs were non-additively expressed, which indicates that miRNAs may participate in heterosis at the jointing stage. miR164, miR1432 and miR528 families were repressed in the four hybrid combinations, and some miRNAs, such as miR156, miR399, and miR395 families, exhibited different expression trends in different hybrid combinations, which may result in varying effects on the heterosis regulatory mechanism.

Conclusions: The potential targets of the identified miRNAs are related to photosynthesis, the response to plant hormones, and nutrient use. Different hybrid combinations employ different mature miRNAs of the same miRNA family and exhibit different expression trends that may result in enhanced or repressed gene expression to regulate heterosis. Taken together, our results reveal a miRNA-mediated network that plays a key role in jointing stage heterosis via posttranscriptional regulation.
\end{abstract}

Keywords: Maize, miRNA, V6 stage, Sixth leaf, Photosynthesis, Jointing stage heterosis

\footnotetext{
* Correspondence: chy9890@163.com

College of Agronomy, State Key Laboratory of Wheat and Maize Crop

Science, Henan Agricultural University, \#15 Longzi Lake University District,

Zhengdong New District, Zhengzhou 450046, Henan, People's Republic of

China
}

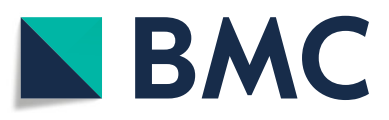

() The Author(s). 2020 Open Access This article is licensed under a Creative Commons Attribution 4.0 International License, which permits use, sharing, adaptation, distribution and reproduction in any medium or format, as long as you give appropriate credit to the original author(s) and the source, provide a link to the Creative Commons licence, and indicate if changes were made. The images or other third party material in this article are included in the article's Creative Commons licence, unless indicated otherwise in a credit line to the material. If material is not included in the article's Creative Commons licence and your intended use is not permitted by statutory regulation or exceeds the permitted use, you will need to obtain permission directly from the copyright holder. To view a copy of this licence, visit http://creativecommons.org/licenses/by/4.0/. The Creative Commons Public Domain Dedication waiver (http://creativecommons.org/publicdomain/zero/1.0/) applies to the data made available in this article, unless otherwise stated in a credit line to the data. 


\section{Background}

Heterosis is a phenomenon in which the vigour of a hybrid is superior to that of both of its homozygous parental lines; heterosis can be used to describe hybrid performance [1] and has played an important role in highproducing and high-quality agricultural products during the past century [2]. While its influence is indispensable in both plant and animal breeding, however, the molecular basis of heterosis is still unclear. Two major hypotheses concerning allelic heterozygosity were put forward to interpret the hereditary basis of heterosis: dominance $[3,4]$ and overdominance [5]. With the rapid development of genomic tools, nonallelic gene interactions have been intensively explored and are also considered a reason for heterosis; therefore, epistasis [6] and gene expression regulatory network hypotheses have been proposed. In recent years, different global expression trends [7, 8], specific protein functions, differential accumulation as well as posttranscriptional modification [9-11] have been identified as being related to heterosis of different organs and at different development stages at the molecular level.

Maize production represents the successful commercialized use of heterosis. Meanwhile, classification of heterotic groups improves the heterosis utilization efficiency; for example, Reid $\times$ Tang-SPT is a major heterotic pattern in China. Furthermore, a leading hybrid developed with the Reid $\times$ Tang-SPT pattern, Zhengdan 958, which has been widely planted across China during the last 20 years, has reached an estimated planting area of more than 43 million hectares to date. In addition, maize is also an appropriate model crop species for exploring the genetic mechanism of heterosis because this species includes many phenotypic, allelic [12], transcriptional $[13,14]$ and translational variations [15] and because its genomic information has been obtained [12, $16,17]$. The jointing stage of maize is a critical stage during which maize transitions from the vegetative stage to the reproductive stage and may affect development at later stages; thus, jointing stage heterosis can reflect grain yield heterosis to some extent. Plant leaves are sites where crucial biological functions occur, for instance, photosynthesis, respiration, transpiration and guttation [18]; in addition, compared with small leaves, larger leaves will give rise to increased photosynthesis yield, water- and nutrient-use efficiency, and biomass productivity [19]. Transcriptional and physiological metabolic processes were found to differ between superhybrid rice and its parents, and the differentially expressed genes were significantly enriched in photosynthesis and carbon fixation [20, 21]. Comparing B73 $\times$ Mo17 with parental inbred lines, the gene with consistent high-parental or above high-parental pattern in at least two tissues was significantly enriched in photosynthesis [22]. All of these studies indicate that photosynthesis plays a key role in heterosis.

miRNAs are approximately 21-nt-long noncoding RNAs that negatively regulate gene expression at the posttranscriptional level [23]. Compared with the translational inhibitory component, miRNA-mediated cleavage of target messenger RNA (mRNA) seems to be the primary mechanism of posttranscriptional regulation [24]. Because of the advent of high-throughput sequencing technology, many miRNAs have been found in diverse plant species on a genome-wide scale, showing tissue-specific and/or development-dependent expression patterns [25-27]. In maize, multiple developmental processes are controlled by miRNA-mediated gene regulation; for instance, miR166 has been reported to regulate leaf polarity [28], overexpression of miR156 results in decreased expression levels of miR172 in the control of juvenile development [29], and miR172 regulates sex determination by targeting APETALA2 floral homeotic transcription factors (TFs) [30]. In addition, many studies have focused on the relationship between miRNAs and heterosis. Research has shown that the expression of some miRNAs in hybrid rice was compared with that in its parents and significant differences were identified [31]. In tomato, miRNA transcriptomes of seedlings of cultivated and wild species and their hybrids have been obtained [32]. A study in maize showed that in hybrid, miR167 is expressed at higher levels in 10day-post-pollination kernels than in its parents, which suggested that miRNAs may participate in the regulation of heterosis [33]. Furthermore, most conserved miRNAs were more abundant in the parental inbred lines than in the hybrids, which implied that miRNAs in hybrids are generally repressed and may be responsible for heterosis of germinating maize embryos [34]. A study of multiple tissues or development stage from eight inbred parents and 12 hybrid genotypes revealed variation and inheritance pattern in sRNAs [35]. Together, all studies indicate that miRNAs play a key role in heterosis; nevertheless, how miRNAs and potential target genes function in jointing stage heterosis of maize is still unclear. In our study, we sought to elucidate the role of miRNAs and their interaction with their target genes in jointing stage heterosis of maize hybrids using next-generation sequencing technology by performing a miRNA transcriptomic analysis of the sixth leaf of four hybrid combinations and parental inbred lines at the V6 stage.

\section{Results}

Photosynthesis index of the sixth leaf at the V6 stage and grain yield of hybrid combinations and inbred lines

The four hybrid combinations were developed in accordance with the same heterotic pattern used in China. To assess the photosynthesis of these hybrid combinations and their parental lines, we measured the leaf area 
(Fig. 1a) and net photosynthesis rate (Fig. 1b) of the sixth leaf at the V6 stage when the sixth leaf had just fully expanded in the field. Furthermore, the grain field (Fig. 1c) was measured after harvest. The results showed that all hybrid combinations performed better than their parental inbred lines did, and each material presented distinctly different photosynthesis rates. We were interested in the potential miRNA regulatory network underlying jointing stage heterosis at the molecular level, so the miRNAs of the four hybrid combinations developed with the Reid $\times$ Tang-SPT pattern and their parental inbred lines were sequenced in the present study.

\section{miRNAs detected in the maize hybrid combinations and inbred lines}

sRNA pools of the sixth leaf at the V6 stage of the four hybrid combinations and inbred lines were sequenced via Illumina sequencing. After the low-quality reads were removed from the raw data, the clean data were further analysed. Analysis of the sRNA length distributions (Fig. 2) in the eight maize samples indicated peak sizes of $21 \mathrm{nt}$ and $24 \mathrm{nt}$, and the sRNAs in the different categories were annotated (Table 1 ).

With the exception of the sRNAs with unannotated sequences, sRNAs were annotated mainly as "rRNA" and "exon sense". In total, there were 182 identified known miRNAs in miRBase (Fig. 3; Additional file 1: Table S1), and 52 novel miRNAs were identified (Additional file 2: Table S2). The number of reads for each miRNA was calculated and normalized to the number of transcripts per million (TPM). The expression pattern in the hybrid combinations was notably different from that in their parental lines.

\section{Expression pattern classification of the differentially expressed miRNAs in the sixth leaf at the V6 stage between the hybrid combinations and inbred lines} To classify the miRNAs into different categories, the miRNAs whose expression significantly differed between the hybrid combinations and parental lines were first identified according to the thresholds of $P<0.01$ and $\log _{2}$ (fold change) $>1$ or $<-1$. Twenty-six and 25 (9 in common) miRNAs were significantly differentially expressed between Zhengdan 958 and Zheng 58 as well as Zhengdan 958 and Chang 7-2, respectively (Fig. 4a; Additional file 3: Table S3-1). When Anyu 5 to Ye 478 and Chang 7-2 were compared, 33 and 21 (4 in common) miRNAs, respectively, were significantly differentially expressed (Fig. 4b; Additional file 4: Table S4-1), and 21 and 29 (11 in common) miRNAs were significantly differentially expressed between Ye $478 \times$ Huangzaosi and Ye 478 and Huangzaosi, respectively (Fig. 4c; Additional file 5: TableS5-1). Similarly, 25 and 31 (9 in common) miRNAs were significantly differentially expressed between Zheng $58 \times$ Huangzaosi and Huangzaosi and Zheng 58, respectively (Fig. 4d; Additional file 6: Table S6-1).

A union of differentially expressed miRNAs in hybrid combination versus maternal inbred line and in hybrid combination versus paternal inbred line was further analysed. These 42, 50, 39 and 47 differentially expressed miRNAs in Zhengdan 958, Anyu 5, Ye $478 \times$ Huangzaosi and Zheng $58 \times$ Huangzaosi were classified into five distinct expression patterns according to the standard of classification mentioned in the methods; accordingly, these miRNAs are hereafter referred to as scaled miRNAs. The expression patterns could be explained by the additive, dominance, and overdominance hypotheses; most of the scaled miRNAs were non-additively (dominantly and over-dominantly) expressed. In total, 17, 23, 18 , and 21 scaled miRNAs were induced (D/A $>0$ ), while 25 , 27,21 , and 26 were repressed $(\mathrm{D} / \mathrm{A}<0)$ in Zhengdan 958, Anyu 5, Ye $478 \times$ Huangzaosi, and Zheng $58 \times$ Huangzaosi, respectively, according to their $\mathrm{D} / \mathrm{A}$ value. In general, miRNAs with low parental expression (-) constituted the most abundant miRNA group in Zhengdan 958, Anyu 5 and Zheng $58 \times$ Huangzaosi; conversely, those with additive expression (+-) in Ye $478 \times$ Huangzaosi constituted the most

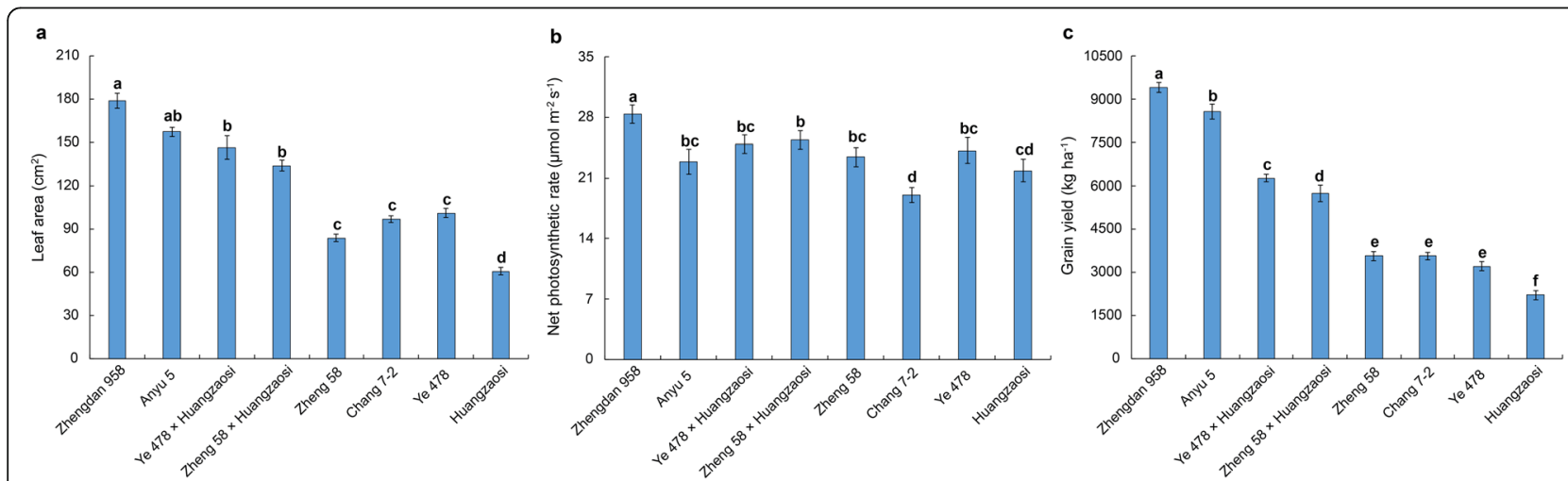

Fig. 1 Characteristics of the leaf area, net photosynthesis rate of the sixth leaf at the V6 stage and grain yield of hybrid combinations and inbred lines. The lowercase letters indicate significant differences at $P<0.05$ (least significant difference) 


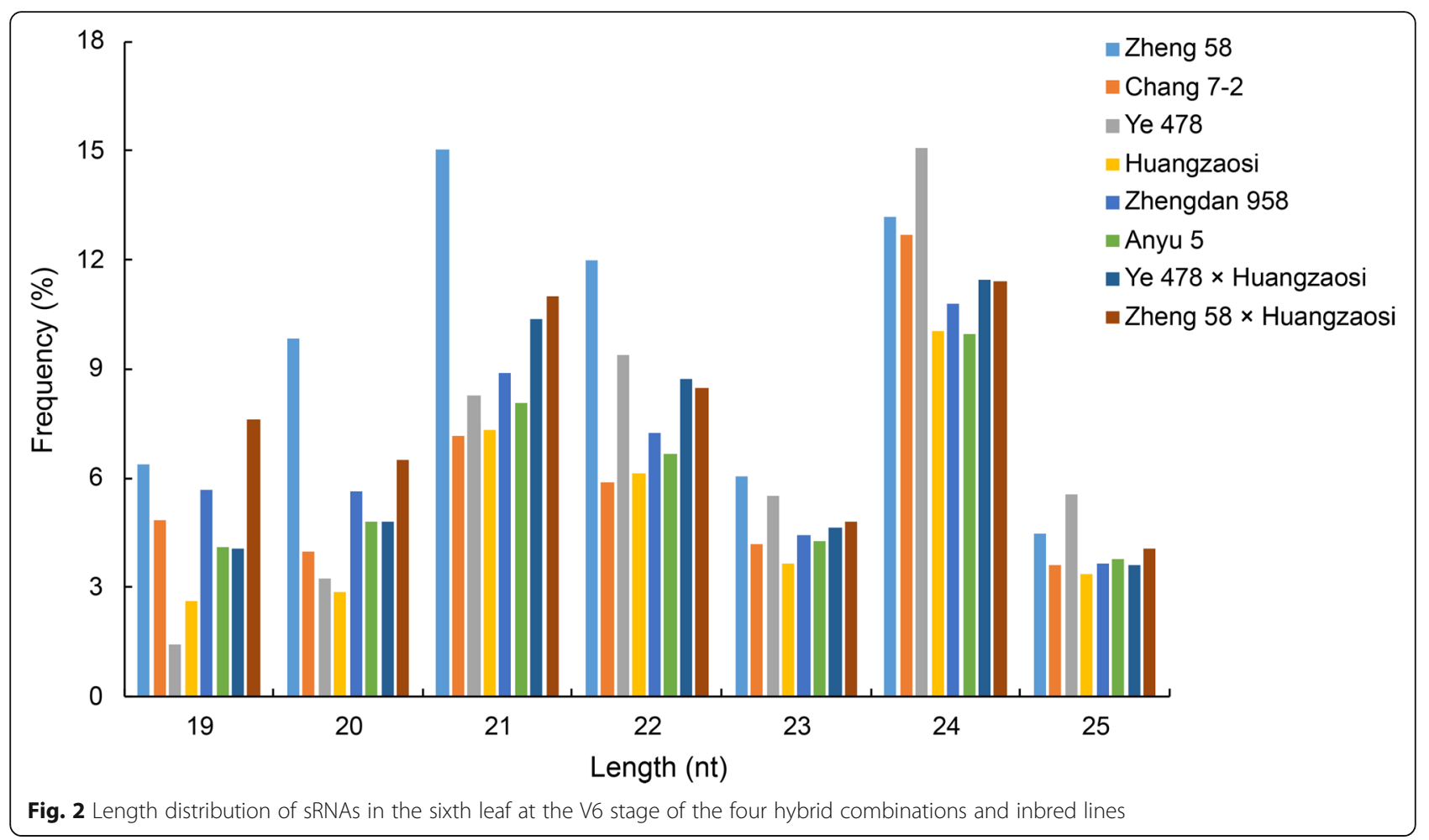

abundant miRNA group (Fig. 4e). According to the statistical data, $60,54,54$, and $55 \%$ of the differentially expressed miRNAs were repressed in Zhengdan 958, Anyu 5, Ye $478 \times$ Huangzaosi, and Zheng $58 \times$ Huangzaosi, respectively. Within the biological process category, the major GO terms for the target genes of these scaled miRNAs in Zhengdan 958 (Fig. 5a), Anyu 5 (Fig. 5b), Ye $478 \times$ Huangzaosi (Fig. 5c), and Zheng $58 \times$ Huangzaosi (Fig. 5d) were enriched in photosynthesis, energy metabolism, chloroplast activity, and response to hormones, which indicates that these biological processes may contribute to heterosis.

\section{Characteristics of the scaled miRNAs in the sixth leaf at the V6 stage among the four hybrid combinations}

There were 13 scaled miRNAs co-detected in the four hybrid combinations (Fig. 4f; Table 2), and some of these scaled miRNAs, including miR160b-3p, miR162-3p,

Table 1 The classification and annotation of small RNAs in inbred lines and hybrid combinations

\begin{tabular}{|c|c|c|c|c|c|c|c|c|c|c|c|c|c|c|c|c|}
\hline \multirow[t]{2}{*}{ Type } & \multicolumn{2}{|l|}{ Ye 478} & \multicolumn{2}{|c|}{ Zheng 58} & \multicolumn{2}{|c|}{ Chang 7-2 } & \multicolumn{2}{|c|}{ Huangzaosi } & \multicolumn{2}{|c|}{$\begin{array}{l}\text { Zhengdan } \\
958\end{array}$} & \multicolumn{2}{|l|}{ Anyu 5} & \multicolumn{2}{|c|}{$\begin{array}{l}\text { Zheng } 58 \times \\
\text { Huangzaosi }\end{array}$} & \multicolumn{2}{|c|}{$\begin{array}{l}\text { Ye } 478 \times \\
\text { Huangzaosi }\end{array}$} \\
\hline & No. & (\%) & No. & (\%) & No. & (\%) & No. & (\%) & No. & (\%) & No. & (\%) & No. & (\%) & No. & $(\%)$ \\
\hline exon antisense & 36,845 & 0.9 & 32,679 & 1.01 & 29,745 & 0.88 & 32,504 & 1.08 & 32,529 & 1 & 29,376 & 0.94 & 31,911 & 1.02 & 35,795 & 1.1 \\
\hline exon sense & 351,662 & 8.63 & 310,750 & 9.6 & 267,915 & 7.91 & 252,898 & 8.41 & 281,694 & 8.69 & 363,730 & 11.62 & 318,596 & 10.22 & 246,757 & 7.6 \\
\hline $\begin{array}{l}\text { intron } \\
\text { antisense }\end{array}$ & 65,219 & 1.6 & 58,022 & 1.79 & 53,706 & 1.59 & 50,573 & 1.68 & 54,489 & 1.68 & 49,937 & 1.59 & 53,732 & 1.72 & 58,784 & 1.81 \\
\hline intron sense & 116,696 & 2.86 & 111,391 & 3.44 & 89,864 & 2.65 & 97,169 & 3.23 & 107,592 & 3.32 & 103,050 & 3.29 & 107,009 & 3.43 & 102,996 & 3.17 \\
\hline miRNA & 1391 & 0.03 & 1720 & 0.05 & 1347 & 0.04 & 1476 & 0.05 & 1530 & 0.05 & 1529 & 0.05 & 1646 & 0.05 & 1684 & 0.05 \\
\hline rRNA & 362,479 & 8.89 & 279,587 & 8.64 & 341,718 & 10.09 & 299,149 & 9.95 & 340,457 & 10.5 & 286,987 & 9.17 & 303,380 & 9.73 & 302,534 & 9.32 \\
\hline repeat & 13,748 & 0.34 & 11,972 & 0.37 & 11,471 & 0.34 & 10,243 & 0.34 & 11,117 & 0.34 & 9977 & 0.32 & 10,564 & 0.34 & 11,587 & 0.36 \\
\hline snRNA & 11,233 & 0.28 & 10,058 & 0.31 & 11,230 & 0.33 & 11,567 & 0.38 & 11,788 & 0.36 & 12,166 & 0.39 & 11,238 & 0.36 & 11,754 & 0.36 \\
\hline snoRNA & 7598 & 0.19 & 7956 & 0.25 & 6524 & 0.19 & 7109 & 0.24 & 6330 & 0.2 & 6972 & 0.22 & 6376 & 0.2 & 6635 & 0.2 \\
\hline tRNA & 122,738 & 3.01 & 68,283 & 2.11 & 103,502 & 3.06 & 90,753 & 3.02 & 86,469 & 2.67 & 79,262 & 2.53 & 72,217 & 2.32 & 82,897 & 2.55 \\
\hline $\begin{array}{l}\text { unannotated } \\
\text { sequences }\end{array}$ & $\begin{array}{l}2,987 \\
131\end{array}$ & 73.27 & $\begin{array}{l}2,343 \\
364\end{array}$ & 72.42 & $\begin{array}{l}2,470 \\
517\end{array}$ & 72.93 & $\begin{array}{l}2,154 \\
506\end{array}$ & 71.63 & $\begin{array}{l}2,307 \\
998\end{array}$ & 71.19 & $\begin{array}{l}2,188 \\
282\end{array}$ & 69.88 & $\begin{array}{l}2,201 \\
162\end{array}$ & 70.6 & $\begin{array}{l}2,384 \\
698\end{array}$ & 73.46 \\
\hline
\end{tabular}




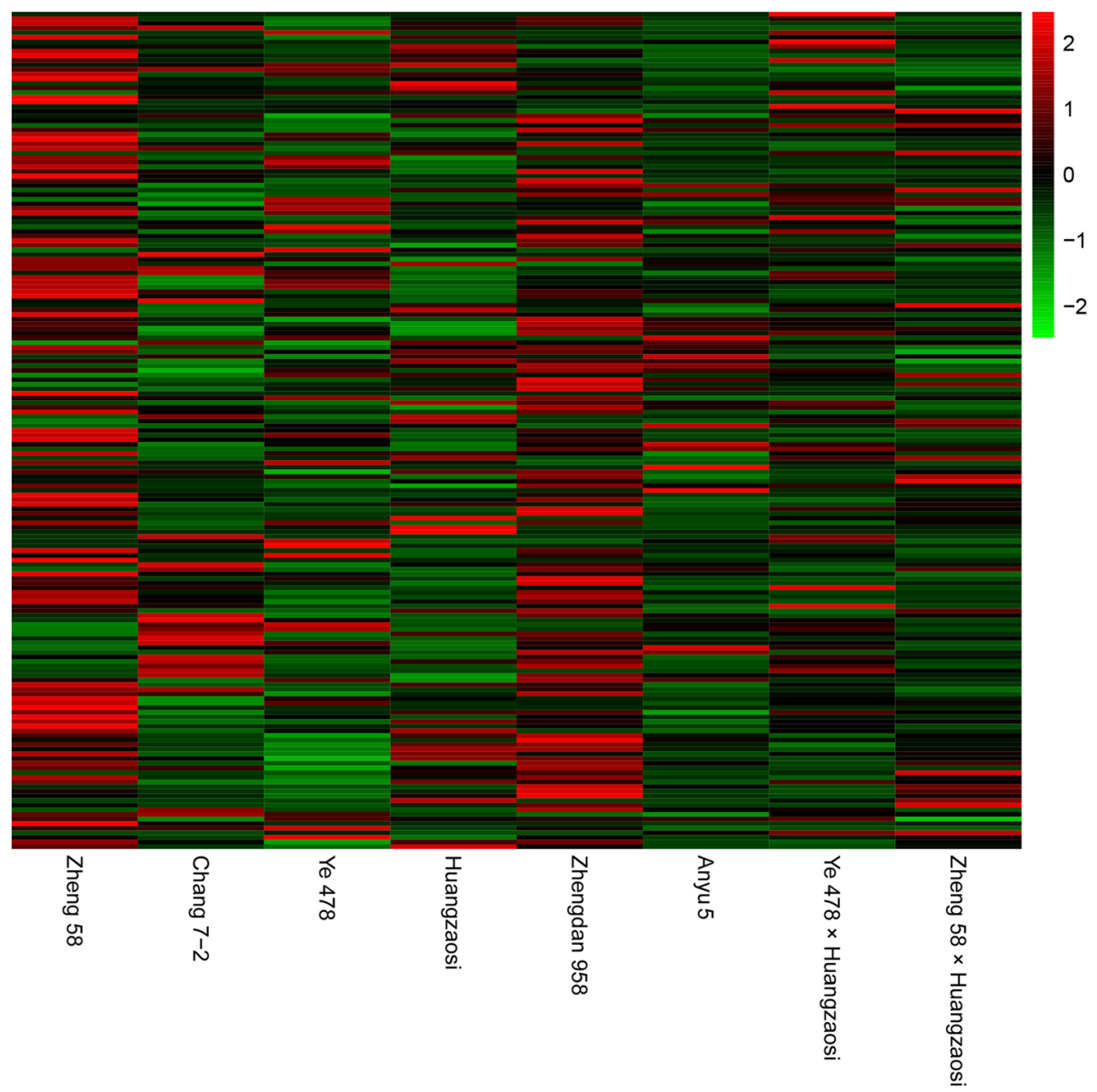

Fig. 3 Expression of the total identified known miRNAs in the four hybrid combinations and the four inbred lines

miR162-5p, miR169c-3p and miR171g-5p, exhibited the same expression pattern in the four hybrid combinations.

Six, 10, 4, and 11 scaled miRNAs were uniquely present in Zhengdan 958, Anyu 5, Ye $478 \times$ Huangzaosi, and Zheng $58 \times$ Huangzaosi (Fig. 4f). Some miRNAs exhibited interesting expression trends. Many miR156 members were differentially expressed in the hybrids, and different miR156 members were expressed in different hybrids. In Zhengdan 958, Anyu 5 and Zheng $58 \times$ Huangzaosi, these members were mainly low parental or extremely low parental expressed, while most of the miR156 members c Ye $478 \times$ Huangzaosi, with the exception of miR156j-3p, which had extremely low parental expression (Table 3). The miR395 members also displayed different expression biases in the different hybrid combinations. The scaled miR395b-3p was presented only in Zhengdan 958, Anyu 5 and Ye $478 \times$ Huangzaosi; scaled miR395b-3p exhibited a "-" expression pattern in the first two hybrid combinations but showed a "+" expression pattern in the last combination (Table 4). miR395e-5p was uniquely present in Zhengdan 958 and exhibited a "+-" expression pattern, zma-
miR395o-5p exhibited a "+-" expression trend in Zheng $58 \times$ Huangzaosi and Ye $478 \times$ Huangzaosi, and zmamiR395a-5p exhibited different expression patterns in all four hybrid combinations.

miR408a uniquely showed a "+" expression pattern in Zhengdan 958, while miR408b-5p uniquely exhibited a "--" expression pattern in Zhengdan 958 and Anyu 5. miRNA528a-5p was repressed in Anyu 5 and Ye $478 \times$ Huangzaosi, while miR528a-3p exhibited a "-" expression pattern in Zhengdan 958 and Zheng $58 \times$ Huangzaosi (Table 5). Members of the miR399 family were mainly induced in the four hybrid combinations (Table 6). Thus, based on these findings, the different expression bias of members from the same miRNA family may also be responsible for heterosis.

\section{Validation of selected miRNAs and their target genes identified via degradome analysis}

Validating target genes is very important for understanding the biological function of miRNAs. In our research, we constructed a degradome library of the sixth leaf at the V6 stage. Target genes identified matched with 


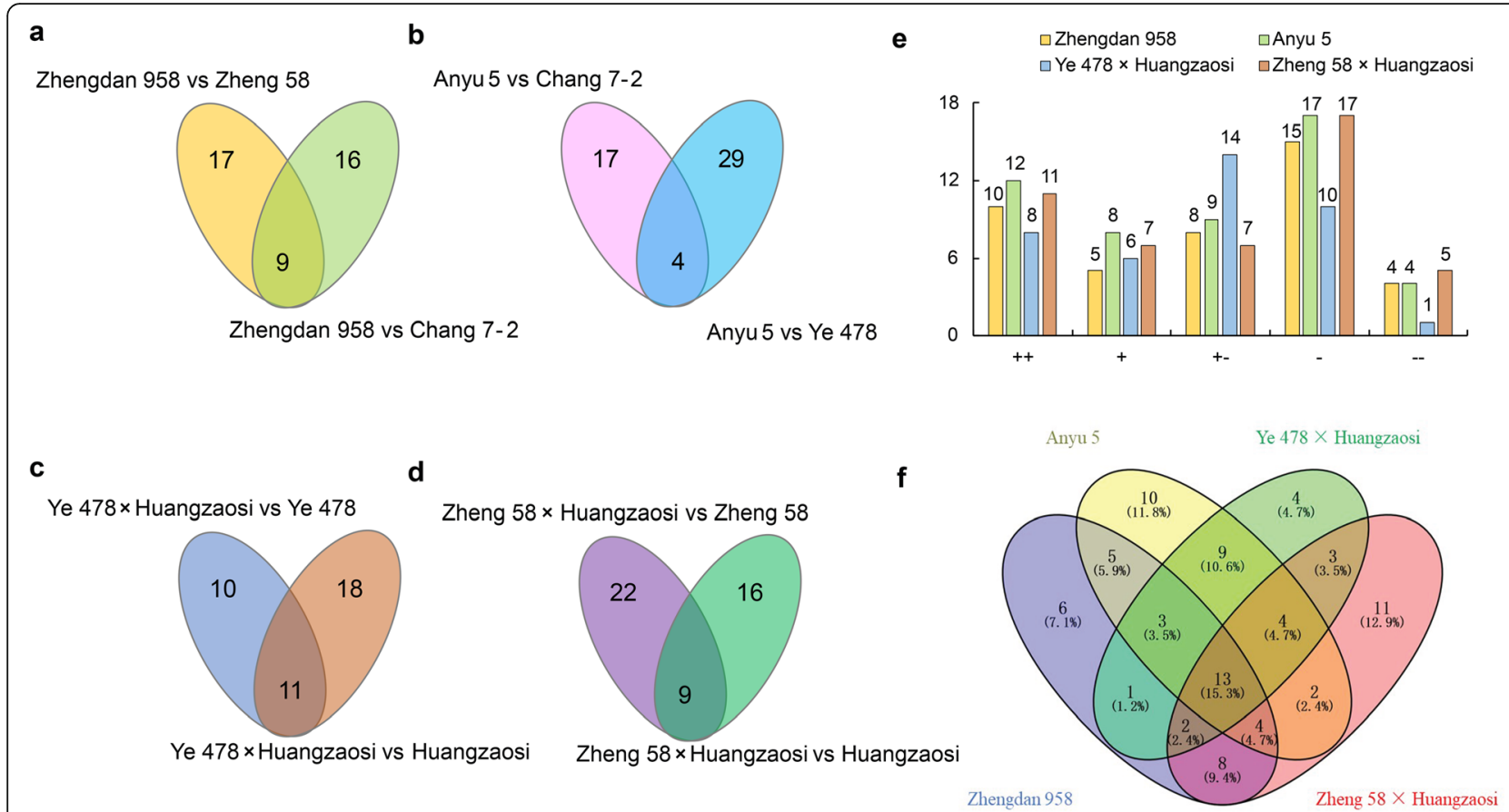

Fig. 4 Characteristics of differentially expressed miRNAs between hybrid combinations and their parental lines and classification of the differentially expressed miRNAs. a-d Venn diagram of differentially expressed miRNAs between the four hybrid combinations and parental lines. e Expression pattern classification of the differentially expressed miRNAs in the four hybrid combinations. " ++ ", extremely high parental expression; " + ", high parental expression; "+-", additive expression; "-", low parental expression; "--", extremely low parental expression. f Venn diagram of the expression patterns of scaled miRNAs in the four hybrid combinations

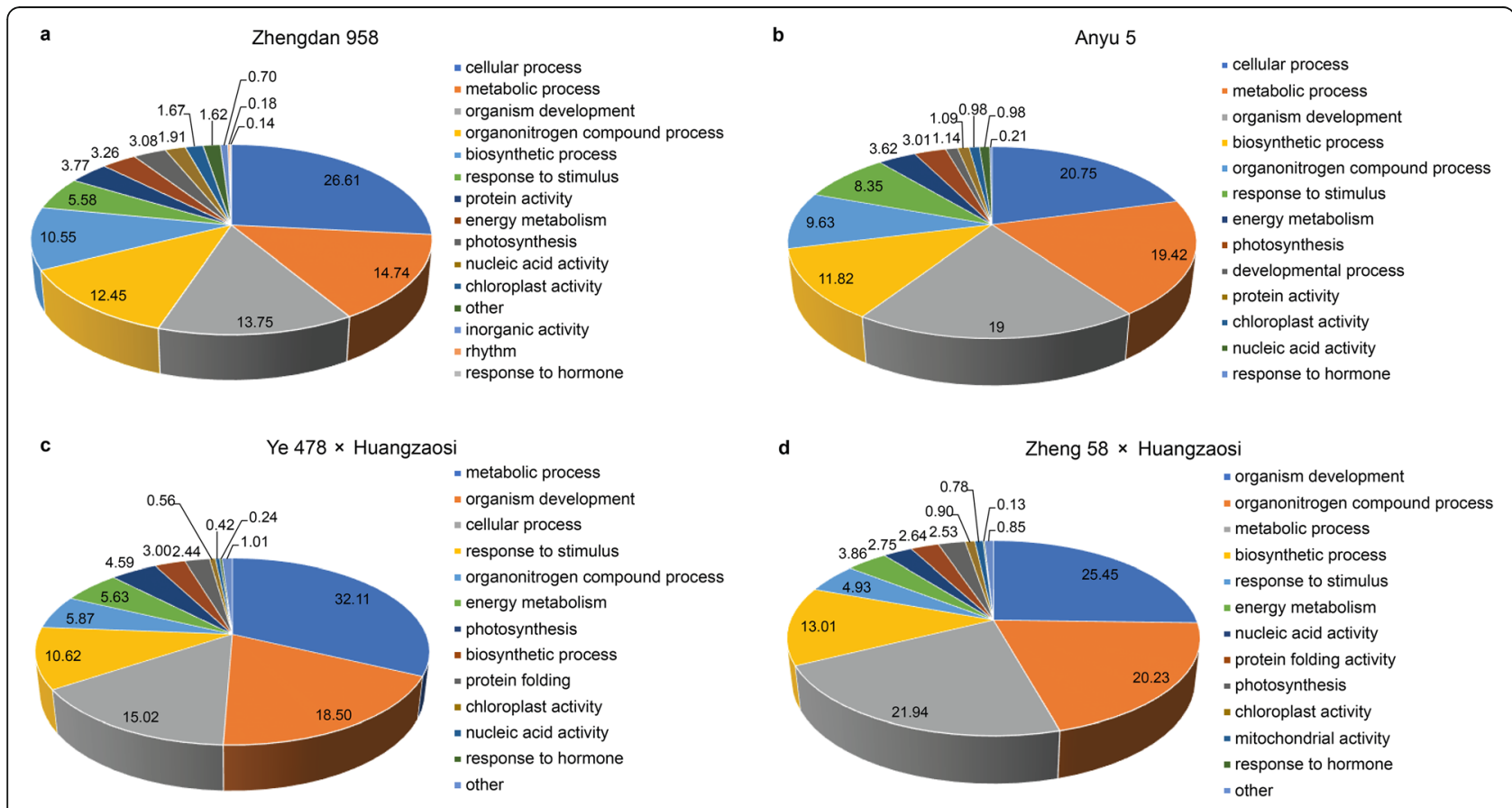

Fig. 5 Major GO categories of biological processes for the target genes identified by the degradome of the scaled miRNAs. a Zhengdan 958; $\mathbf{b}$ Anyu 5; c Ye $478 \times$ Huangzaosi; d Zheng $58 \times$ Huangzaosi 
Table 2 Expression pattern of the commonly expressed scaled miRNAs of the four hybrid combinations

\begin{tabular}{|c|c|c|c|c|c|c|c|c|}
\hline \multirow[t]{2}{*}{ miRNA ID } & \multicolumn{2}{|c|}{ Zhengdan 958} & \multicolumn{2}{|c|}{ Anyu 5} & \multicolumn{2}{|c|}{ Zheng $58 \times$ Huangzaosi } & \multicolumn{2}{|c|}{ Ye $478 \times$ Huangzaosi } \\
\hline & $\mathrm{D} / \mathrm{A}$ & classification & $\mathrm{D} / \mathrm{A}$ & classification & $\mathrm{D} / \mathrm{A}$ & classification & $\mathrm{D} / \mathrm{A}$ & classification \\
\hline zma-miR160b-3p & 8.36 & ++ & 2.69 & ++ & 3.26 & ++ & 2.62 & ++ \\
\hline zma-miR166b-5p & 5.38 & ++ & -0.6 & - & 12.99 & ++ & 0.34 & +- \\
\hline zma-miR171g-5p & 18.79 & ++ & 58.05 & ++ & 10.14 & ++ & 27.09 & ++ \\
\hline zma-miR169c-3p & 1.67 & + & 1.9 & + & 0.9 & + & 1.44 & + \\
\hline zma-miR399d-5p & 1.08 & + & 8.35 & ++ & 0.41 & +- & 2.87 & ++ \\
\hline zma-miR162-3p & -0.11 & +- & -0.22 & +- & -0.2 & +- & 0.41 & +- \\
\hline zma-miR164e-5p & -0.39 & +- & -0.65 & - & -0.4 & +- & -0.38 & +- \\
\hline zma-miR169f-3p & 0.1 & +- & 0.74 & + & 0.33 & +- & 0.36 & +- \\
\hline zma-miR397b-3p & -0.47 & +- & -0.21 & +- & -0.8 & - & 0.51 & + \\
\hline zma-miR167j-3p & -1.06 & - & -0.36 & +- & -0.34 & +- & 1.43 & + \\
\hline zma-miR162-5p & -0.82 & - & -0.74 & - & -0.82 & - & -0.53 & - \\
\hline zma-miR395a-5p & -0.57 & - & -2.14 & - & 2.33 & ++ & -0.19 & +- \\
\hline zma-miR1432-5p & -5.36 & - & -0.92 & - & -3.71 & - & -1.14 & - \\
\hline
\end{tabular}

Note: The D/A was calculated according to the formula (F1-MP)/(HP-MP) by expression abundance; "++", extremely high parental expression (D/A greater than 2); "+", high parental expression (D/A greater than 0.5 and below 2); “+-", additive expression (D/A greater than - 0.5 and below 0.5$)$; "-, low parental expression (D/ A greater than -2 and below -0.5$)$; “--", extremely low parental expression (D/A below -2 )

scaled miRNAs in Zhengdan 958 (Additional file 3: Table S3-2), Anyu 5 (Additional file 4: Table S4-2), Ye $478 \times$ Huangzaosi (Additional file 5: Table S5-2), and Zheng $58 \times$ Huangzaosi (Additional file 6: Table S6-2). As may be expected, most transcripts targeted by conserved miRNAs were relative to conserved target genes. For instance, miR156a-5p targeted SPL11, miR164e-5p targeted NAC79, miR395b-3p targeted sulfate transporter 2.2, and miR528a-5p targeted cupredoxin superfamily proteins (Fig. 6). The expression profiles of these conserved miRNAs and target genes identified by the degradome were determined for the four hybrid combinations and their parental lines. We performed reverse transcription reactions for the total RNA, followed by quantitative real-time polymerase chain reaction (qRTPCR). The internal control ZmActin was employed for
qRT-PCR, and the $2^{-\Delta \Delta C t}$ method was applied for expression-level calculation. The expression profiles of selected miRNAs were largely consistent with those from the sequencing data. Compared with the corresponding miRNAs, the target genes showed an opposite expression trend (Fig. 7; Additional file 7, 8, 9: Fig. S1, S2, S3).

\section{Discussion}

\section{Relationships between heterosis, photosynthesis, and miRNAs}

Heterosis, the superior performance of F1s relative to their parents, is prevalent for many traits and crosses of maize [36]. Maize displays dramatic heterosis for numerous traits, such as biomass, height, root growth, photosynthesis, starch metabolism, grain yield and biotic/

Table 3 Expression pattern of miR156 family members in four hybrid combinations

\begin{tabular}{|c|c|c|c|c|c|c|c|c|}
\hline \multirow[t]{2}{*}{ miRNA ID } & \multicolumn{2}{|c|}{ Zhengdan 958} & \multicolumn{2}{|c|}{ Anyu 5} & \multicolumn{2}{|c|}{ Zheng $58 \times$ Huangzaosi } & \multicolumn{2}{|c|}{ Ye $478 \times$ Huangzaosi } \\
\hline & $\mathrm{D} / \mathrm{A}$ & classification & $\bar{D} / \mathrm{A}$ & classification & $\mathrm{D} / \mathrm{A}$ & classification & $\mathrm{D} / \mathrm{A}$ & classification \\
\hline zma-miR156e-3p & 1 & 1 & 1 & 1 & -5.62 & - & 1 & 1 \\
\hline zma-miR156 h-3p & -0.86 & - & 1 & 1 & 1 & 1 & 1 & 1 \\
\hline zma-miR156k-5p & -0.99 & - & 1 & 1 & 1 & 1 & 1 & 1 \\
\hline zma-miR156d-3p & -6.63 & - & 1 & 1 & -1.56 & - & 1 & 1 \\
\hline zma-miR156l-3p & 1 & 1 & 1 & 1 & -0.75 & - & -0.35 & +- \\
\hline zma-miR156j-3p & 1 & 1 & -3.19 & - & -1.61 & - & -5.11 & - \\
\hline zma-miR156a-5p & -1.12 & - & 1 & 1 & 1 & $\backslash$ & 0.26 & +- \\
\hline
\end{tabular}

Note: The D/A was calculated according to the formula (F1-MP)/(HP-MP) by expression abundance; "++", extremely high parental expression (D/A greater than 2); "+", high parental expression (D/A greater than 0.5 and below 2); "+-", additive expression (D/A greater than -0.5 and below 0.5 ); “-, low parental expression (D/A greater than -2 and below -0.5$) ;$ "--", extremely low parental expression

(D/A below - 2) 
Table 4 Expression pattern of miR395 family members in four hybrid combinations

\begin{tabular}{|c|c|c|c|c|c|c|c|c|}
\hline \multirow[t]{2}{*}{ miRNA ID } & \multicolumn{2}{|c|}{ Zhengdan 958} & \multicolumn{2}{|c|}{ Anyu 5} & \multicolumn{2}{|c|}{ Zheng $58 \times$ Huangzaosi } & \multicolumn{2}{|c|}{ Ye $478 \times$ Huangzaosi } \\
\hline & $\mathrm{D} / \mathrm{A}$ & classification & $\mathrm{D} / \mathrm{A}$ & classification & $\mathrm{D} / \mathrm{A}$ & classification & $\mathrm{D} / \mathrm{A}$ & classification \\
\hline zma-miR395e-5p & -0.3 & +- & 1 & 1 & 1 & 1 & 1 & 1 \\
\hline zma-miR395a-5p & -0.57 & - & -2.14 & - & 2.33 & ++ & -0.19 & +- \\
\hline zma-miR395o-5p & 1 & 1 & 1 & 1 & 0.18 & +- & -0.06 & +- \\
\hline zma-miR395b-3p & -1.02 & - & -1.41 & - & 1 & 1 & 0.69 & + \\
\hline
\end{tabular}

Note: The D/A was calculated according to the formula (F1-MP)/(HP-MP) by expression abundance; "++", extremely high parental expression (D/A greater than 2): " + ", high parental expression (D/A greater than 0.5 and below 2); "+-", additive expression (D/A greater than -0.5 and below 0.5$)$; "-", low parental expression (D/A greater than -2 and below -0.5$) ; "-{ }^{-\prime}$, extremely low parental expression (D/A below - 2)

abiotic stress resistance; hence, maize has served as a model plant for the study of heterosis. Zhengdan 958, a commercial hybrid that has a large planting area in China, represents the successful use of the Reid $\times$ Tang-SPT heterotic pattern. Recently, molecular and physiological proof has suggested that photosynthesis is associated with heterosis [37-39]. In Arabidopsis, whether in hybrid combinations or in their parents, if the rate of photosynthesis was constant per unit leaf, the hybrids presented an increase in total photosynthesis capability because of increased numbers of chloroplasts per cell, cell size and leaf area [19]. In maize, there are many studies regarding how miRNAs function in heterosis, such as studies pertaining to seed germination, internode expansion, and endosperm development [33, 40, 41]. We are interested in the potential mechanism by which miRNAs function in Zhengdan 958, Anyu 5 and the other two hybrid combinations of the Reid $\times$ Tang-SPT heterotic pattern to regulate jointing stage heterosis. Understanding this mechanism can help us better understand heterosis.

Speculative miRNA-mRNA regulatory mechanism involved in jointing stage heterosis

In the present study, 182 known miRNAs were identified from the eight materials at the V6 stage, and the miRNAs differentially expressed in hybrids versus at least one parental line were scaled according to their D/A values. The expression of $25,27,21$, and 26 miRNAs was repressed, while the expression of $17,23,18$, and 21 miRNAs was induced, in Zhengdan 958, Anyu 5, Ye $478 \times$ Huangzaosi, and Zheng $58 \times$ Huangzaosi, respectively. Interactions between these scaled miRNAs and target genes may be related to heterosis. For instance, miR156, which is a class of star miRNAs reportedly involved in plant development and growth by binding specifically to the GTAC cis-element, target the SQUAMOSA promoter-binding-like (SPL) family of TFs $[42,43]$. Given the increased knowledge regarding miR156, the miR156/SPL module has been suggested to be a multifaceted tool with which to enhance agronomic traits [44]. In rice, the os-miR156 and OsSPL14 modules control ideal plant architecture [45], and SPL TFs have also been found to modulate grain size, grain quality, panicle branching as well as plant height [46, 47]. In maize, miR156 was repressed in the hybrids, which may have induced SPL expression to increase internode expansion [41]. In our study, the repression of miR156a-5p may cause overexpression of Squamosa promoterbinding-like protein 11 to accelerate the phase transition, which indicates that the hybrids have stronger growth potential than the parental inbred lines.

In maize inbred line B73, miR408b-5p has a singlenucleotide polymorphism (SNP) $(\mathrm{A} / \mathrm{G})$ at the 11 th base position from the 5 'end that differs from that of Mo17, which has been confirmed in another study, showing that parental expression between the B73 $\times$ Mo17 and Mo17 $\times$ B73 reciprocal crosses may cause functional

Table 5 Expression pattern of miR408 and miR528 family members in four hybrid combinations

\begin{tabular}{|c|c|c|c|c|c|c|c|c|}
\hline \multirow[t]{2}{*}{ miRNA ID } & \multicolumn{2}{|c|}{ Zhengdan 958} & \multicolumn{2}{|c|}{ Anyu 5} & \multicolumn{2}{|c|}{ Zheng $58 \times$ Huangzaosi } & \multicolumn{2}{|c|}{ Ye $478 \times$ Huangzaosi } \\
\hline & $\mathrm{D} / \mathrm{A}$ & classification & $\mathrm{D} / \mathrm{A}$ & classification & $\mathrm{D} / \mathrm{A}$ & classification & $\mathrm{D} / \mathrm{A}$ & classification \\
\hline zma-miR408b-5p & -8.08 & - & -6.08 & - & 1 & 1 & 1 & 1 \\
\hline zma-miR408a & 1.38 & + & 1 & 1 & 1 & 1 & 1 & 1 \\
\hline zma-miR528a-5p & 1 & 1 & -2.96 & - & 1 & 1 & -1.09 & - \\
\hline zma-miR528a-3p & -1.72 & - & 1 & 1 & -0.93 & - & 1 & $\backslash$ \\
\hline
\end{tabular}

Note: The D/A was calculated according to the formula (F1-MP)/(HP-MP) by expression abundance; "++", extremely high parental expression (D/A greater than 2); "+", high parental expression (D/A greater than 0.5 and below 2); "+-", additive expression (D/A greater than - 0.5 and below 0.5 ); "-, low parental expression (D/ A greater than -2 and below - 0.5); “--", extremely low parental expression (D/A below -2 ) 
Table 6 Expression pattern of miR399 family members in four hybrid combinations

\begin{tabular}{|c|c|c|c|c|c|c|c|c|}
\hline \multirow[t]{2}{*}{ miRNA ID } & \multicolumn{2}{|c|}{ Zhengdan 958} & \multicolumn{2}{|c|}{ Anyu 5} & \multicolumn{2}{|c|}{ Zheng $58 \times$ Huangzaosi } & \multicolumn{2}{|c|}{ Ye $478 \times$ Huangzaosi } \\
\hline & $\mathrm{D} / \mathrm{A}$ & classification & $\mathrm{D} / \mathrm{A}$ & classification & $\mathrm{D} / \mathrm{A}$ & classification & $\mathrm{D} / \mathrm{A}$ & classification \\
\hline zma-miR399e-3p & 1 & 1 & 0.08 & +- & 1 & 1 & 1 & 1 \\
\hline zma-miR399c-5p & 1 & 1 & 1 & 1 & 1 & 1 & -0.06 & +- \\
\hline zma-miR399d-5p & 1.08 & + & 8.35 & ++ & 0.41 & +- & 2.87 & ++ \\
\hline zma-miR399e-5p & 13.25 & ++ & 1 & 1 & 4.52 & ++ & 1 & 1 \\
\hline zma-miR399d-3p & 1 & 1 & 0.52 & + & 1 & 1 & -0.33 & +- \\
\hline zma-miR399a-3p & 1 & 1 & 1.87 & + & 1 & 1 & -0.39 & +- \\
\hline zma-miR399b-3p & 1 & 1 & 1.47 & + & 1 & 1 & -0.78 & - \\
\hline zma-miR399f-3p & 1 & 1 & 1.08 & + & 1 & 1 & -0.39 & +- \\
\hline zma-miR399 g-3p & 33.41 & ++ & 6.16 & ++ & 1 & 1 & 1 & 1 \\
\hline zma-miR399a-5p & 11.18 & ++ & 5.39 & ++ & 1 & 1 & 2.06 & ++ \\
\hline zma-miR399j-5p & 5.61 & ++ & 1.62 & + & 17.75 & ++ & 1 & 1 \\
\hline zma-miR399i-5p & 1 & 1 & -0.91 & - & 0.63 & + & -0.66 & - \\
\hline zma-miR399b-5p & 1 & 1 & 2.37 & ++ & -1.08 & - & -0.72 & - \\
\hline
\end{tabular}

Note: The D/A was calculated according to the formula (F1-MP)/(HP-MP) by expression abundance; "++", extremely high parental expression (D/A greater than 2); "+", high parental expression (D/A greater than 0.5 and below 2); “+-", additive expression (D/A greater than - 0.5 and below 0.5$)$; “-", low parental expression (D/ A greater than -2 and below -0.5 ); “--", extremely low parental expression (D/A below -2 )

variation [40]. The target genes of miR408b-5p identified by degradome, early light-induced proteins (ELIPs), which is a light-harvesting complex that binds chlorophyll and takes in solar energy in green plants in Arabidopsis. Suppression of the fast accumulation of ELIPs under intense light stress leads to leaf decolouration and causes extended photooxidative damage [48]. In our study, only the Mo17-allele miR408b-5p was downregulated in Zhengdan 958 and Anyu 5. Interestingly, miR408a, a miRNA that has been proven to result in improved biomass and seed yield by overexpression in Arabidopsis [49], was uniquely induced in Zhengdan 958. MIR408-overexpressing Arabidopsis plants presented remarkably improved leaf area, petiole length, plant height, flower size, and silique length as well as a significantly increased photosynthesis rate, which led to more biomass and seed yield. The genes whose expression was downregulated in MIR408-OX transgenic plants were remarkably enriched in pathways involving ribosomes, photosynthesis, carbon fixation, as well as pigment biosynthesis processes. In another study, miR408 overexpression resulted in increased fresh weight and increased root length at the seedling stage, and at the adult stage, all the leaves were enlarged [50]. In maize, miR408a upregulated in response to chilling stress to maintain leaf growth [51]. Based on our research, the high parental expression exhibited by miR408a only in Zhengdan 958 may also lead to increased photosynthesis and, thus, may have an effect on heterosis of maize hybrid combinations.

Sulfur is a mineral element essential to plant growth and is involved in photosynthesis, respiration, nitrogen and carbohydrate metabolism, thus regulating plant development [52]. Sulfate is the main form of inorganic sulfur available to plants; it is absorbed by plant roots and then translocated to diverse tissues for assimilation. In Arabidopsis, there are two forms of mature miR395 (miR395a,d,e and miR395b,c,f), which target two families of genes, the ATP sulfurylase and the sulfate transporter 2;1, both of which are involved in the sulfate metabolism pathway, and their transcripts are repressed strongly in miR395-overexpressing transgenic Arabidopsis, which have a relatively small stature and are slightly more chlorotic than the wild-type plants are. The mechanism involved in the maintenance of sulfate homeostasis by miR395 in plants has been elucidated [53]. Thus, we can infer that miR395b-3p is downregulated in hybrids to take advantage of sulfate more effectively.

Phosphate plays a key role in plant growth and development as an essential macronutrient, and it is a key structural component of nucleic acids, phospholipids, and the energy-carrying molecule ATP [54, 55]. miR399 family members participated in phosphate homeostasis as a phosphate deficiency-responsive factor in Arabidopsis. miR399 was shown to be induced under $\mathrm{Pi}$ deprivation to upregulate the splicing of the target mRNA PHO2 and suppress downstream PHT1 and PHO1 ubiquitination degradation by $\mathrm{PHO} 2$, promoting $\mathrm{Pi}$ absorption and transport [56-60]. In maize, miR399 expression is upregulated in response to Pi deficiency, and overexpression of miR399b leads to P accumulation in the shoots [61]. miR399 is also upregulated in the elongation zone, which may play possible roles in switching from cell division to cell elongation during leaf 

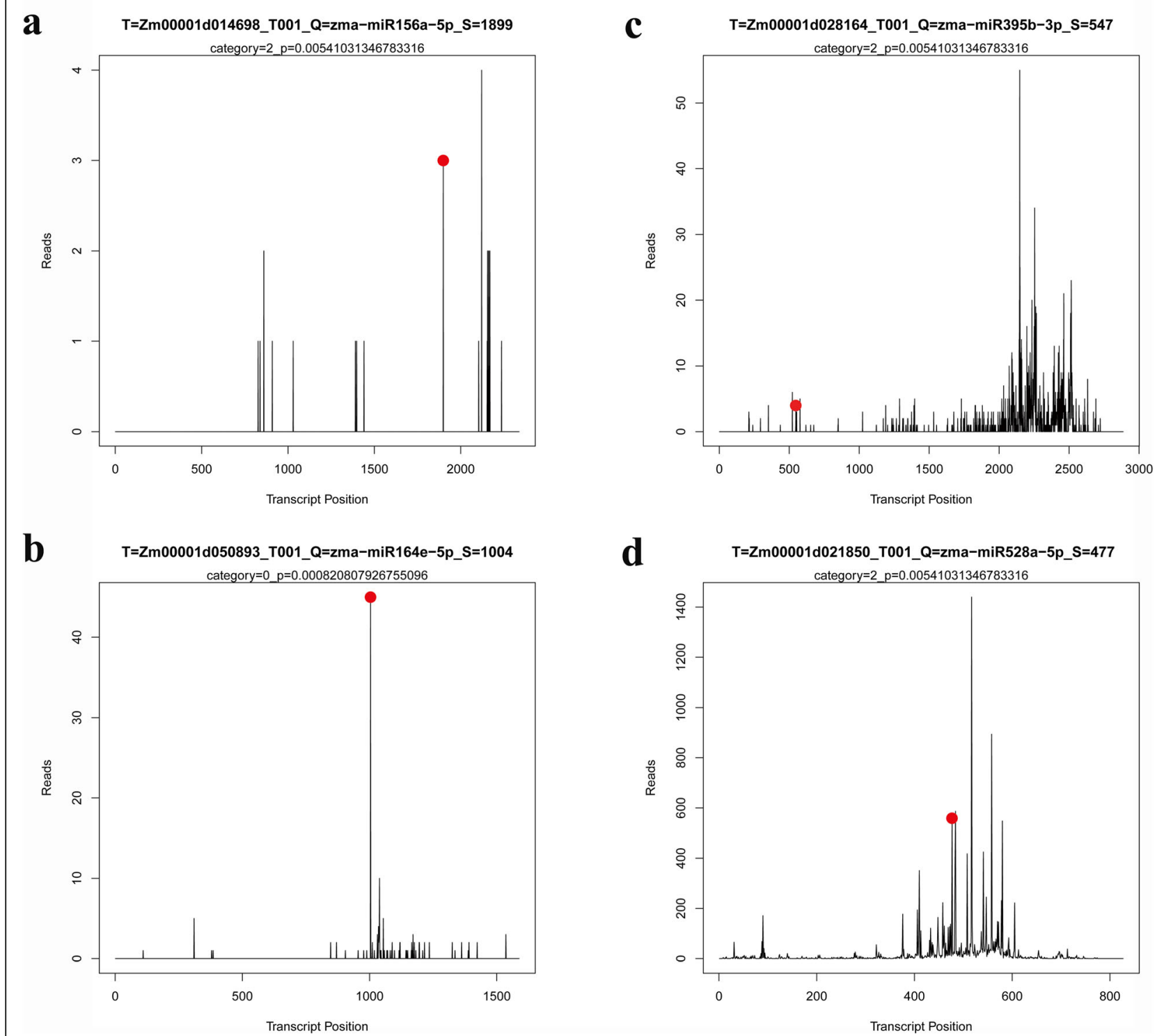

Fig. 6 Examples of T-plots of miRNA targets confirmed by degradome sequencing. he T-plots show the distribution of the degradome reads along the full length of the target mRNA. The red point indicates the cleavage site of each transcript. a The cleavage features in SPL11 (Zm00001d014698_T001) mRNA by miR156a-5p. b Cleavage features in NAC domain-containing protein 79 (Zm00001d050893_T001) mRNA by miR164e-5p. c Cleavage features in sulfate transporter 2.2 (Zm00001d028164_T001) mRNA by miR395b-3p. d Cleavage features in a cupredoxin superfamily protein member (Zm00001d021850_T001) mRNA by miR528a-5p

development [62]. In our study, miR399 was induced in hybrids, which may improve P-use efficiency to accelerate growth and development, which may ultimately be a cause of heterosis.

miR528s are a class of miRNAs restricted to monocots [63]. In rice, miR528 was proven to target at least four mRNA transcripts of genes that encode two plastocyaninlike proteins, an L-ascorbate oxidase and an EIN3-binding F-box protein [64]. $\mathrm{Cu}$ is an important element for protein synthesis [65]. There is an important class of $\mathrm{Cu}$ containing proteins that function primarily as electron transfer proteins rather than as oxidases, which are commonly named blue $\mathrm{Cu}$ proteins or cupredoxins, such as plastocyanin-like proteins, which play an important role during photosynthesis [66]. In our study, miR528a-5p was repressed in the hybrid combinations, which may increase electron transfer to regulate photosynthesis.

The target of miR164 is the TF-encoding gene NAC1, which binds specifically to the cis-element IDE2. In many plant species, NAC TFs increase resistance to biotic and abiotic stresses by regulating the auxin signalling pathway [67-69]. Auxin can also promote NAC1 


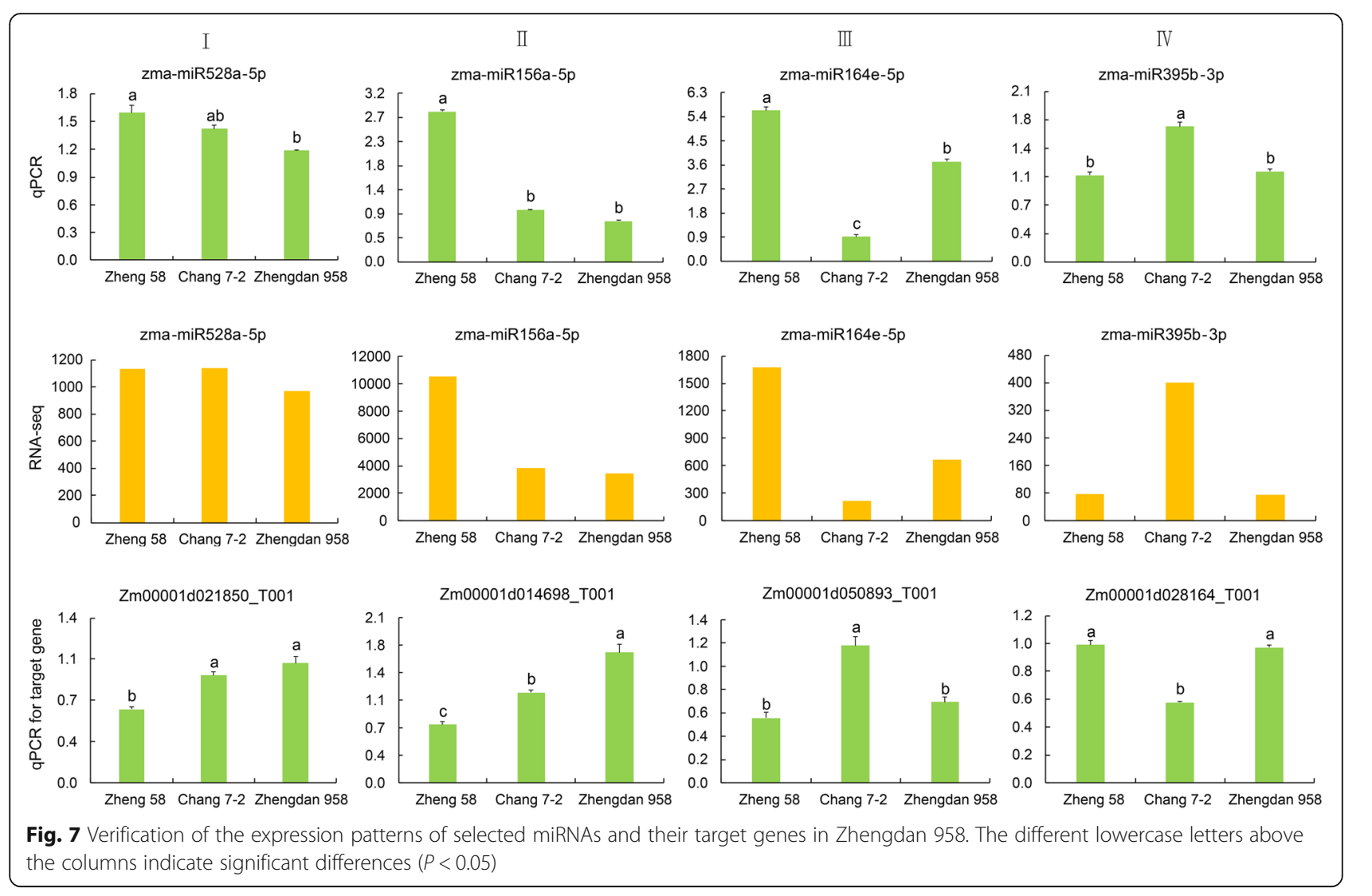

expression and functions downstream of TIR1 [70]; both auxin-induced miR164 and ubiquitination can decrease NAC1 transcription to reduce auxin signalling [71]. In our study, miR164e-5p was downregulated in the hybrid combinations; thus, NAC1 transcription may be upregulated, resulting in increased auxin signalling.

PS1-F (photosystem 1-F subunit) is a target of miR1432 identified by degradome in our study and has been demonstrated to regulate grain yield and tiller number in rice. Chlorophyll concentration and the electron transport rate were notably reduced in the homozygous Ds insertion OsPS1-F mutant line, leading to a reduction in plant height, tiller number, and grain yield, as well as pale yellow leaf coloration. If the mutant was complemented by proUBI::OsPS1-F, the phenotype due to the mutation was less pronounced [72]. In our study, miR1432-5p was repressed in the hybrids, inducing PS1$\mathrm{F}$, as a result, photosynthesis increased.

The miRNA-mRNA modules may regulate jointing stage heterosis (Fig. 8). Among these miRNAs, the miR1432, miR164, and miR528 families were repressed in the four hybrid combinations; however, interestingly, the miR408, miR395, miR399, and miR156 families were expressed as different mature miRNA forms in the different hybrids and may exhibit different expression trends, which we believe may have different regulatory effects. Although many studies have worked on heterosis from different levels, such as gene expression, presence/ absence variation, DNA methylation changes, protein changes as well as metabolite changes, our study focus on the miRNAs' behaviour in hybrids and parents, which provide new knowledge about miRNA-mediated regulatory mechanism on heterosis. However, heterosis is a complicated, integrated scientific problem which still needs further studied.

\section{Conclusions}

In this study, we found that 85 miRNAs were differentially expressed between the four hybrid combinations and parental lines at the V6 stage, and the union of the differentially expressed miRNAs was classified according to their D/A value. Of these scaled miRNAs, the expression of 25, 27, 21, and 26 miRNAs was repressed, while the expression of 17, 23, 18, and 21 miRNAs was found to be induced in Zhengdan 958, Anyu 5, Ye $478 \times$ Huangzaosi, and Zheng $58 \times$ Huangzaosi, respectively. Most of the scaled miRNAs were non-additively expressed. The scaled miRNAs from the same family expressed in different mature miRNAs exhibited different patterns in the different hybrid combinations, which may result in different posttranscriptional regulatory mechanism effects, followed by various heterosis 


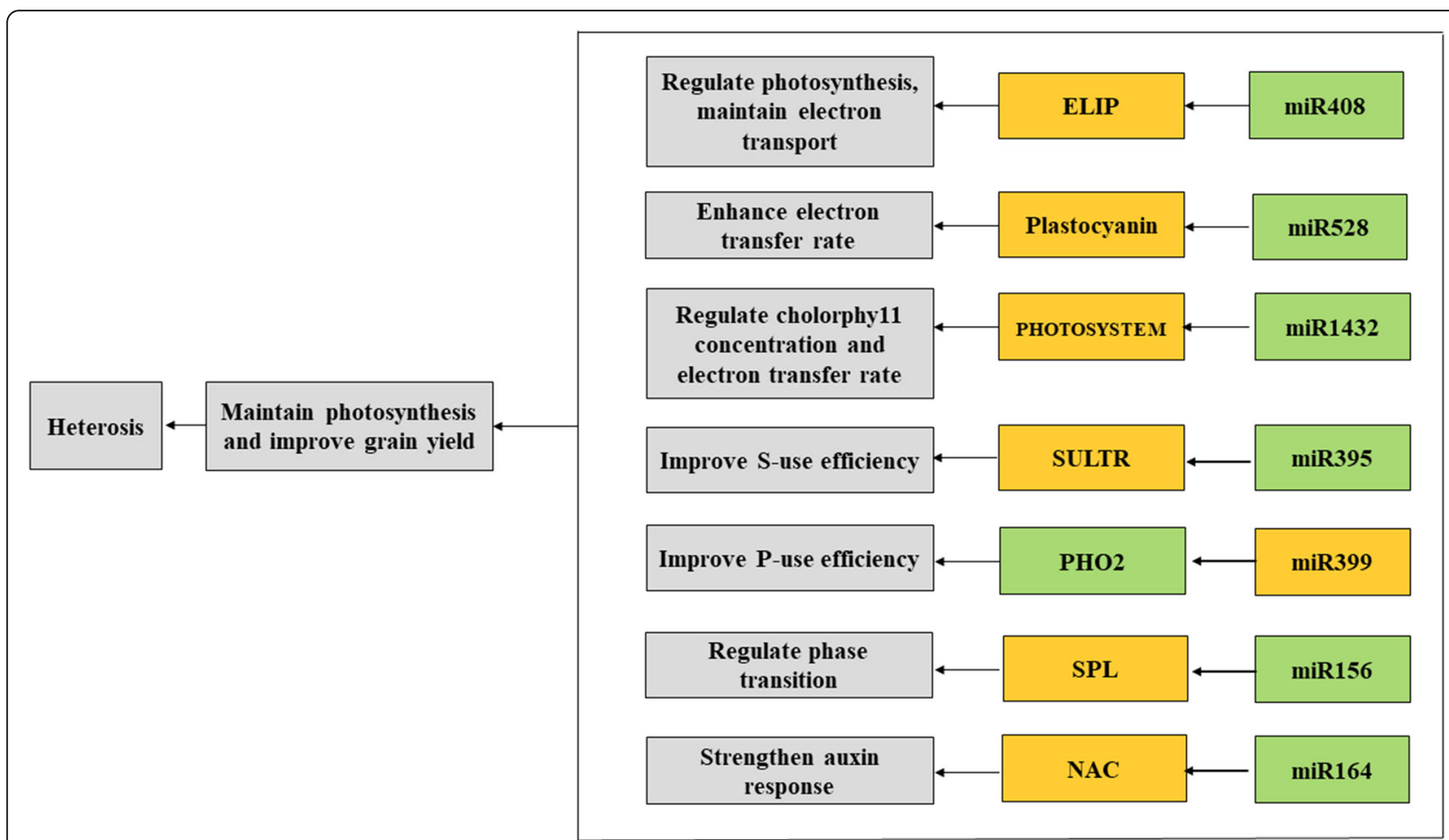

Fig. 8 Model for miRNAs and their target genes associated with photosynthesis, hormone effects, and nutrient use. The black letters in the yellow block indicate upregulation, and the black letters in the green block indicate downregulation. The black arrows represent the direction of regulation. ELIP: early light-induced protein; SULTR, sulfate transporter; PHO2, PHOSPHATE 2; SPL, SQUAMOSA promoter-binding-like family transcription factors; NAC1, NAC domain-containing protein

phenotypes. We also validated the expression of some representative miRNAs by $\mathrm{qRT}-\mathrm{PCR}$. The potential miRNA target genes were confirmed via degradome sequencing. Our results indicate that a miRNA-mediated posttranscriptional regulation network, these scaled miRNAs (miR408, miR528, miR1432, miR395, miR399, miR156, miR164), may have roles in jointing stage heterosis via photosynthesis regulation. Our work provides useful information for further exploration of mechanisms involved in heterosis by miRNAs.

\section{Methods}

\section{Plant materials}

Zhengdan 958 (Zheng $58 \times$ Chang 7-2), Anyu 5 (Ye $478 \times$ Chang 7-2), Ye $478 \times$ Huangzaosi, and Zheng $58 \times$ Huangzaosi were developed in accordance with the popular heterotic pattern Reid $\times$ Tang-SPT in China, and their parental lines (Zheng 58 and Ye 478 are famous inbred lines from the domestic Reid group, and Chang 7-2 and Huangzaosi were famous inbred lines from the Tang-SPT group) were used as materials. We crossed the Zheng 58 and Ye 478 (maternal lines) with Chang 7-2, Huangzaosi (paternal lines) to gain four hybrid combinations, and the four hybrid combinations as well as the four inbred lines were then planted in the summer of 2018 on a farm at Henan Agricultural University (Zhengzhou, $113^{\circ} 42^{\prime} \mathrm{E}, 34^{\circ} 48^{\prime} \mathrm{N}$ ) in northern China. In each replication, forty-five seeds of each material were planted in pots arranged in three lines in the field with $0.25-\mathrm{m}$ line spacing and $0.60-\mathrm{m}$ row spacing, such that the plant density was $67,500 \mathrm{ha}^{-1}$, and there were three replications.

\section{Photosynthesis index and grain yield measurements}

When plants reached the V6 stage, the sixth leaf had just fully expanded, photosynthesis of the sixth leaf of 10 randomly selected seedlings was assessed in each replication for the eight materials by an LI-6400 photosynthesis system (LI-COR Biosciences, Lincoln, NE, USA) in the field between 8:00 a.m. and 11:00 a.m. Then, for the above-mentioned 10 selected seedlings, the length and greatest width of the sixth-fully-expanded leaf were used to measure leaf area according to the method of Montgomery [73]. In each replication, the middle row of each material was harvested to measure kernel weight per ear, and grain yield was calculated by the following formula: Grain yield $(\mathrm{kg}$ $\left.\mathrm{ha}^{-1}\right)=$ kernel weight per ear $(\mathrm{kg}) \times$ plant density $(67$, $500 \mathrm{ha}^{-1}$ ). Three replications were applied. 


\section{Maize RNA extraction, sRNA sequencing and miRNA identification}

In each biological replication, equal amounts of tissue from the mid-part of the fully-expanded sixth leaf of 5 randomly selected seedlings from each material were harvested as soon as the plants reached the V6 stage and the sixth leaf had just fully expanded. They were stored at $-80^{\circ} \mathrm{C}$, and three replicates were obtained. Total RNA from each material was extracted using TRIzol reagent, and 24 sRNA libraries were constructed at Beijing Genomics Institute.

The raw data from Illumina sequencing were processed to filter and remove low-quality, repeats and lowcomplexity reads. To identify conserved miRNAs, unique sRNA sequences with lengths between 18 and $25 \mathrm{nt}$ were mapped to miRNAs reported in miRBase 21.0 (http://www.mirbase.org/). After conserved miRNAs were annotated, the remaining parts of small RNA reads were used to identify novel miRNAs by the prediction software MIREAP (http://sourceforge.net/projects/mireap/). sRNAs that could map to the maize genome were considered potential miRNA candidates only if meeting the strict criteria reported in the literature [74].

\section{Identification of differentially expressed miRNAs and expression pattern classifications}

The expression levels were normalized as TPM values. To evaluate statistical significance, a $t$-test was applied. Differentially expressed miRNAs between hybrid combinations and parental lines were identified with the following criteria: $P$-value was $<0.01$ and $\log _{2}$ (fold change) $>1$ or $<-1$. For each differentially expressed miRNA, the scaled difference was calculated as dominance/additivity (D/A value), based on TPM; the degree of dominance was calculated as hybrid - mid-parent; and the degree of additivity was calculated as high-parent - midparent. The D/A value was calculated by the formula: (F1-MP)/(HP-MP) [22]. A D/A greater than zero was considered induced, while a D/A less than zero was considered repressed. Scaled miRNA expression patterns were divided into five groups: $(1)$ " ++ ", extremely high parental expression, with a D/A greater than 2; (2) “+”, high parental expression, with a D/A greater than 0.5 and less than 2; (3) "+-", additive expression, with a D/A greater than -0.5 and less than 0.5 ; (4) “-”, low parental expression, with a D/A greater than -2 and less than 0.5; and (5) “--", extremely low parental expression, with a D/A less than - 2 [34].

Degradome library construction and target identification We equally mixed the 24 total RNAs used for miRNA sequencing to construct a single degradome library according to the method reported in the literature [75]. Single-end sequencing $(50 \mathrm{bp})$ was performed on an
Illumina HiSeq 2000 (Illumina, San Diego, CA USA). We used CleaveLand 3.0 to analyse the generated sequencing data. The identified target genes of the corresponding differentially expressed miRNAs were annotated with GO terms (http://www.geneontology.org/), which were considered significantly enriched when their $P$-value was $<0.05$.

\section{Quantification of selected miRNAs and their target genes using qRT-PCR}

We used a One Step PrimeScript miRNA cDNA Synthesis Kit (TaKaRa Co., Tokyo, Japan) to perform reverse transcription reactions according to the manufacturer's instructions. qRT-PCR was performed with a SYBR PrimeScript miRNA RT-PCR Kit in conjunction with a fluorescence detection system (Roche LightCycler 480 II). The expression levels of the miRNAs were calculated using the $2^{-\triangle \Delta C T}$ method (the primer sequences used are given in Additional file 10: Table S10). Differences in expression levels between hybrids and inbred lines were tested according to Fisher's least significant difference test using Statistical Program for Social Science (SPSS) software; $P<0.05$ was considered statistically significant.

\section{Supplementary Information}

The online version contains supplementary material available at https://doi. org/10.1186/s12870-020-02751-3.

Additional file 1: Table S1. Known miRNAs identified in this study.

Additional file 2: Table S2. Novel miRNAs identified in this study.

Additional file 3: Table S3. Expression pattern of differentially expressed miRNAs in Zhengdan 958 and target function identified by degradome.

Additional file 4: Table S4. Expression pattern of differentially expressed miRNAs in Anyu 5 and target function identified by degradome.

Additional file 5: Table S5. Expression pattern of differentially expressed miRNAs in Ye $478 \times$ Huangzaosi and target function identified by degradome.

Additional file 6: Table S6. Expression pattern of differentially expressed miRNAs in Zheng $58 \times$ Huangzaosi and target function identified by degradome.

Additional file 7: Fig. S1. Verification of the expression patterns of selected miRNAs and their target genes in Anyu 5. The different lowercase letters above the columns indicate significant differences

Additional file 8: Fig. S2. Verification of the expression patterns of selected miRNAs and their target genes in Ye $478 \times$ Huangzaosi. The different lowercase letters above the columns indicate significant differences $(P<0.05)$.

Additional file 9: Fig. S3. Verification of the expression patterns of selected miRNAs and their target genes in Zheng $58 \times$ Huangzaosi. The different lowercase letters above the columns indicate significant differences $(P<0.05)$.

Additional file 10: Table S10. Oligonucleotide primers used for qRTPCR assays in this study.

Abbreviations

miRNA: MicroRNA; sRNA: Small RNA; V6 stage: The six-leaf stage; GO: Gene ontology; qRT-PCR: Quantitative reverse transcription-polymerase chain reaction; SPL: Squamosa promoter binding protein-like; NAC: NAC domain- 
containing protein; ELIPs: Early light-induced proteins; PSII: Photosystem II complex; PS1-F: Photosystem-1 F subunit; PHO2: PHOSPHATE 2; PHO1: PHOSPHATE 1; PHT1: Phosphate transporter protein 1; EIN3-binding Fbox: ETHYLENE-INSENSITIVE3 transcription factors binding F-box

\section{Acknowledgements}

We would like to thank Lei Tian, Moubiao Zhang, and Jingyang Gao for their help with the bioinformatics analysis. We also thank American Journal Experts (Durham, North Carolina, USA) for proofreading our manuscript.

\section{Authors' contributions}

LCW, LXK helped design the experiment; YHC designed the experiments; GGH carried out the experiments and wrote the draft; XLM revised the manuscript; YHD and GGH performed the qRT-PCR and prepared some of the figures; FFZ, QNZ, TYL and DDD carried out the field trait analysis and sampling. All authors have contributed to the corrections of the manuscript and approve it for submission.

\section{Funding}

The work was funded by National Key Research and Development Program of China with grant 2016YFD0101205. This funding body has no role in the design of the study; collection, analysis and interpretation of data; and in writing the manuscript.

\section{Availability of data and materials}

The datasets generated and analysed during the current study are available in the NCBI Sequence Read Archive (SRA) database under Bioproject PRJNA649665 (https://www.ncbi.nlm.nih.gov/bioproject/PRJNA649665). The materials (Zheng 58, Ye 478, Chang 7-2, Huangzaosi, Zhengdan 958, Anyu 5, Ye $478 \times$ Huangzaosi, Zheng $58 \times$ Huangzaosi) used during the current study are available from the corresponding author on reasonable request.

\section{Ethics approval and consent to participate}

Collection of plant materials in this study complied with institutional, national or international guidelines.

\section{Consent for publication}

Not applicable.

\section{Competing interests}

The authors declare that they have no competing interests.

Received: 21 June 2020 Accepted: 22 November 2020 Published online: 30 November 2020

\section{References}

1. Shull GH. Beginnings of the heterosis concept. In: Gowen JW, editor. Heterosis. Ames: lowa State College Press; 1952. p. 14-48.

2. Duvick DN. Biotechnology in the 1930s: the development of hybrid maize. Nat Rev Genet. 2001;2(1):69-74.

3. Bruce $A B$. The mendelian theory of heredity and the augmentation of vigor. Science. 1910;32(827):627-8.

4. Jones DF. Dominance of linked factors as a means of accounting for Heterosis. P Natl Acad Sci USA. 1917;3(4):310-2.

5. East EM. Heterosis. Genetics. 1936;21(4):375-97.

6. Powers L. Relative yields of inbred lines and F1 hybrids of tomato. Bot Gaz. 1945;106(3):247-68.

7. Hoecker N, Keller B, Muthreich N, Chollet D, Descombes P, Piepho HP, Hochholdinger F. Comparison of maize (Zea mays L.) F1-hybrid and parental inbred line primary root transcriptomes suggests organ-specific patterns of nonadditive gene expression and conserved expression trends. Genetics. 2008;179(3):1275-83.

8. Hochholdinger $\mathrm{F}$, Hoecker N. Towards the molecular basis of heterosis. Trends Plant Sci. 2007;12(9):427-32.

9. Guo M, Rupe MA, Zinselmeier C, Habben J, Bowen BA, Smith OS. Allelic variation of gene expression in maize hybrids. Plant Cell. 2004;16(7):1707-16.

10. Liu Y, von Behrens I, Muthreich N, Schütz W, Nordheim A, Hochholdinger F. Regulation of the pericycle proteome in maize (Zea mays L.) primary roots by RUM1 which is required for lateral root initiation. Eur J Cell Biol. 2010; 89(2-3):236-41.
11. Paschold A, Marcon C, Hoecker N, Hochholdinger F. Molecular dissection of heterosis manifestation during early maize root development. Theor Appl Genet. 2010;120(2):383-8.

12. Schnable PS, Ware D, Fulton RS, Stein JC, Wei F, Pasternak S, Liang C, Zhang J, Fulton L, Graves TA, et al. The B73 maize genome: complexity, diversity, and dynamics. Science. 2009;326(5956):1112-5.

13. Ma J, Morrow DJ, Fernandes J, Walbot V. Comparative profiling of the sense and antisense transcriptome of maize lines. Genome Biol. 2006;7(3):R22.

14. Stupar RM, Springer NM. Cis-transcriptional variation in maize inbred lines B73 and Mo17 leads to additive expression patterns in the F1 hybrid. Genetics. 2006;173(4):2199-210.

15. Hoecker N, Lamkemeyer T, Sarholz B, Paschold A, Fladerer C, Madlung J, Wurster K, Stahl M, Piepho HP, Nordheim A, et al. Analysis of nonadditive protein accumulation in young primary roots of a maize (Zea mays L.) $F(1)$ hybrid compared to its parental inbred lines. Proteomics. 2008;8(18):3882-94.

16. Li C, Song W, Luo Y, Gao S, Zhang R, Shi Z, Wang X, Wang R, Wang F, Wang $J$, et al. The HuangZaoSi maize genome provides insights into genomic variation and improvement history of maize. Mol Plant. 2019;12(3):402-9.

17. Sun S, Zhou Y, Chen J, Shi J, Zhao H, Zhao H, Song W, Zhang M, Cui Y, Dong $X$, et al. Extensive intraspecific gene order and gene structural variations between Mo17 and other maize genomes. Nat Genet. 2018;50(9):1289-95.

18. Jacotot A, Marchand C, Gensous S, Allenbach M. Effects of elevated atmospheric $\mathrm{CO}_{2}$ and increased tidal flooding on leaf gas-exchange parameters of two common mangrove species: Avicennia marina and Rhizophora stylosa. Photosynth Res. 2018:138(2):249-60.

19. Fujimoto R, Taylor JM, Shirasawa S, Peacock WJ, Dennis ES. Heterosis of Arabidopsis hybrids between C24 and col is associated with increased photosynthesis capacity. P Natl Acad Sci USA. 2012;109(18):7109-14.

20. Wei G, Tao Y, Liu G, Chen C, Luo R, Xia H, Gan Q, Zeng H, Lu Z, Han Y, et al. A transcriptomic analysis of superhybrid rice $L Y P 9$ and its parents. P Natl Acad Sci USA. 2009;106(19):7695-701.

21. Song GS, Zhai HL, Peng YG, Zhang L, Wei G, Chen XY, Xiao YG, Wang L, Chen YJ, Wu B, et al. Comparative transcriptional profiling and preliminary study on heterosis mechanism of super-hybrid rice. Mol Plant. 2010;3(6):1012-25.

22. Zhou P, Hirsch CN, Briggs SP, Springer NM. Dynamic patterns of gene expression Additivity and regulatory variation throughout maize development. Mol Plant. 2019;12(3):410-25.

23. Jones-Rhoades MW, Bartel DP, Bartel B. MicroRNAs and their regulatory roles in plants. Annu Rev Plant Biol. 2006;57:19-53.

24. Brodersen $P$, Sakvarelidze-Achard L, Bruun-Rasmussen M, Dunoyer $P$, Yamamoto $Y Y$, Sieburth L, Voinnet O. Widespread translational inhibition by plant miRNAs and siRNAs. Science. 2008:320(5880):1185-90.

25. Xue LJ, Zhang JJ, Xue HW. Characterization and expression profiles of miRNAs in rice seeds. Nucleic Acids Res. 2009;37(3):916-30.

26. Jiao $Y$, Song W, Zhang M, Lai J. Identification of novel maize miRNAs by measuring the precision of precursor processing. BMC Plant Biol. 2011;11:141.

27. Sun F, Guo G, Du J, Guo W, Peng H, Ni Z, Sun Q, Yao Y. Whole-genome discovery of miRNAs and their targets in wheat (Triticum aestivum L.). BMC Plant Biol. 2014;14:142

28. Juarez MT, Kui JS, Thomas J, Heller BA, Timmermans MC. microRNAmediated repression of rolled leaf1 specifies maize leaf polarity. Nature. 2004;428(6978):84-8

29. Nag A, Jack T. Sculpting the flower; the role of microRNAs in flower development. Curr Top Dev Biol. 2010;91:349-78.

30. Chuck G, Meeley R, Irish E, Sakai H, Hake S. The maize tasselseed4 microRNA controls sex determination and meristem cell fate by targeting Tasselseed6/ indeterminate spikelet1. Nat Genet. 2007;39(12):1517-21.

31. Chen F, He G, He H, Chen W, Zhu X, Liang M, Chen L, Deng XW. Expression analysis of miRNAs and highly-expressed small RNAs in two rice subspecies and their reciprocal hybrids. J Integr Plant Biol. 2010;52(11):971-80.

32. Shivaprasad PV, Dunn RM, Santos BA, Bassett A, Baulcombe DC Extraordinary transgressive phenotypes of hybrid tomato are influenced by epigenetics and small silencing RNAs. EMBO J. 2012;31(2):257-66.

33. Mica E, Gianfranceschi L. Pe' ME. Characterization of five microRNA families in maize. J Exp Bot. 2006;57(11):2601-12.

34. Ding D, Wang Y, Han M, Fu Z, Li W, Liu Z, Hu Y, Tang J. MicroRNA transcriptomic analysis of heterosis during maize seed germination. PLoS One. 2012;7(6):e39578.

35. Crisp PA, Hammond R, Zhou P, Vaillancourt B, Lipzen A, Daum C, Barry K, de Leon N, Buell CR, Kaeppler SM, et al. Variation and inheritance of small RNAs in maize Inbreds and F1 hybrids. Plant Physiol. 2020;182(1):318-31. 
36. Flint-Garcia SA, Buckler ES, Tiffin P, Ersoz E, Springer NM. Heterosis is prevalent for multiple traits in diverse maize germplasm. PLoS One. 2009; 4(10):e7433.

37. Offermann S, Peterhansel C. Can we learn from heterosis and epigenetics to improve photosynthesis? Curr Opin Plant Biol. 2014;19:105-10.

38. Song Y, Zhang Z, Tan X, Jiang Y, Gao J, Lin L, Wang Z, Ren J, Wang X, Qin L, et al. Association of the molecular regulation of ear leaf senescence/stress response and photosynthesis/metabolism with heterosis at the reproductive stage in maize. Sci Rep. 2016;6:29843.

39. Mohayeji M, Capriotti AL, Cavaliere C, Piovesana S, Samperi R, Stampachiacchiere S, Toorchi M, Lagana A. Heterosis profile of sunflower leaves: a label free proteomics approach. J Proteome. 2014;99:101-10.

40. Xin M, Yang G, Yao Y, Peng H, Hu Z, Sun Q, Wang X, Ni Z. Temporal small RNA transcriptome profiling unraveled partitioned miRNA expression in developing maize endosperms between reciprocal crosses. Front Plant Sci. 2015;6:744.

41. Zhao P, Ding D, Zhang F, Zhao X, Xue Y, Li W, Fu Z, Li H, Tang J. Investigating the molecular genetic basis of heterosis for internode expansion in maize by microRNA transcriptomic deep sequencing. Funct Integr Genomics. 2015;15(3):261-70.

42. Wang JW, Park MY, Wang $L$, Koo Y, Chen XY, Weigel D, Poethig RS. miRNA control of vegetative phase change in trees. PLoS Genet. 2011;7(2): e1002012.

43. Wang L, Gu X, Xu D, Wang W, Wang H, Zeng M, Chang Z, Huang H, Cui X. miR396-targeted AtGRF transcription factors are required for coordination of cell division and differentiation during leaf development in Arabidopsis. $J$ Exp Bot. 2011;62(2):761-73.

44. Wang $H$, Wang $H$. The miR156/SPL module, a regulatory hub and versatile toolbox, gears up crops for enhanced agronomic traits. Mol Plant. 2015;8(5): 677-88

45. Jiao Y, Wang Y, Xue D, Wang J, Yan M, Liu G, Dong G, Zeng D, Lu Z, Zhu X, et al. Regulation of OsSPL14 by OsmiR156 defines ideal plant architecture in rice. Nat Genet. 2010;42(6):541-4.

46. Miura K, Ikeda M, Matsubara A, Song XJ, Ito M, Asano K, Matsuoka M, Kitano H, Ashikari M. OSSPL14 promotes panicle branching and higher grain productivity in rice. Nat Genet. 2010;42(6):545-9.

47. Si L, Chen J, Huang X, Gong H, Luo J, Hou Q, Zhou T, Lu T, Zhu J, Shangguan $Y$, et al. OsSPL13 controls grain size in cultivated rice. Nat Genet. 2016:48(4):447-56

48. Hutin C, Nussaume L, Moise N, Moya I, Kloppstech K, Havaux M. Early lightinduced proteins protect Arabidopsis from photooxidative stress. P Natl Acad Sci USA. 2003;100(8):4921-6.

49. Song Z, Zhang L, Wang Y, Li H, Li S, Zhao H, Zhang H. Constitutive expression of miR408 improves biomass and seed yield in Arabidopsis. Front Plant Sci. 2017:8:2114.

50. Zhang H, Li L. SQUAMOSA promoter binding protein-like7 regulated microRNA408 is required for vegetative development in Arabidopsis. Plant J. 2013;74(1):98-109.

51. Aydinoglu F. Elucidating the regulatory roles of microRNAs in maize (Zea mays L.) leaf growth response to chilling stress. Planta. 2020;251(2):38.

52. Smith IK, Lang AL. Translocation of sulfate in soybean (Glycine max L. Merr). Plant Physiol. 1988;86(3):798-802.

53. Liang G, Yang F, Yu D. MicroRNA395 mediates regulation of sulfate accumulation and allocation in Arabidopsis thaliana. Plant J. 2010;62(6): 1046-57.

54. Péret B, Clément M, Nussaume L, Desnos T. Root developmental adaptation to phosphate starvation: better safe than sorry. Trends Plant Sci. 2011;16(8): 442-50.

55. López-Arredondo DL, Leyva-González MA, González-Morales SI, López-Bucio J, Herrera-Estrella L. Phosphate nutrition: improving low-phosphate tolerance in crops. Annu Rev Plant Biol. 2014;65:95-123.

56. Fujii $\mathrm{H}$, Chiou TJ, Lin SI, Aung K, Zhu JK. A miRNA involved in phosphatestarvation response in Arabidopsis. Curr Biol. 2005;15(22):2038-43.

57. Chiou TJ, Aung K, Lin SI, Wu CC, Chiang SF, Su CL. Regulation of phosphate homeostasis by MicroRNA in Arabidopsis. Plant Cell. 2006;18(2):412-21.

58. Lin SI, Chiang SF, Lin WY, Chen JW, Tseng CY, Wu PC, Chiou TJ. Regulatory network of microRNA399 and PHO2 by systemic signaling. Plant Physiol. 2008;147(2):732-46.

59. Liu TY, Huang TK, Tseng CY, Lai YS, Lin SI, Lin WY, Chen JW, Chiou TJ. PHO2dependent degradation of $\mathrm{PHO} 1$ modulates phosphate homeostasis in Arabidopsis. Plant Cell. 2012;24(5):2168-83.
60. Huang TK, Han CL, Lin SI, Chen YJ, Tsai YC, Chen YR, Chen JW, Lin WY, Chen PM, Liu TY, et al. Identification of downstream components of ubiquitinconjugating enzyme PHOSPHATE2 by quantitative membrane proteomics in Arabidopsis roots. Plant Cell. 2013;25(10):4044-60.

61. Du Q, Wang K, Zou C, Xu C, Li WX. The PILNCR1-miR399 regulatory module is important for low phosphate tolerance in maize. Plant Physiol. 2018; 177(4):1743-53

62. Aydinoglu F, Lucas SJ. Identification and expression profiles of putative leaf growth related microRNAs in maize (Zea mays L.) hybrid ADA313. Gene. 2019;690:57-67.

63. Liu B, Li P, Li X, Liu C, Cao S, Chu C, Cao X. Loss of function of OsDCL1 affects microRNA accumulation and causes developmental defects in rice. Plant Physiol. 2005;139(1):296-305.

64. Yang R, Li P, Mei H, Wang D, Sun J, Yang C, Hao L, Cao S, Chu C, Hu S, et al. Fine-tuning of MiR528 accumulation modulates flowering time in Rice. Mol Plant. 2019;12(8):1103-13.

65. Pilon M, Abdel-Ghany SE, Cohu CM, Gogolin KA, Ye H. Copper cofactor delivery in plant cells. Curr Opin Plant Biol. 2006;9(3):256-63.

66. Joliot $\mathrm{P}$, Joliot A. Cyclic electron flow in C3 plants. Biochim Biophys Acta. 2006;1757(5-6):362-8.

67. Tran LS, Nakashima K, Sakuma Y, Simpson SD, Fujita Y, Maruyama K, Fujita M, Seki M, Shinozaki K, Yamaguchi-Shinozaki K. Isolation and functional analysis of Arabidopsis stress-inducible NAC transcription factors that bind to a drought-responsive cis-element in the early responsive to dehydration stress 1 promoter. Plant Cell. 2004;16(9):2481-98.

68. Christianson JA, Wilson IW, Llewellyn DJ, Dennis ES. The low-oxygeninduced NAC domain transcription factor ANAC102 affects viability of Arabidopsis seeds following low-oxygen treatment. Plant Physiol. 2009; 149(4):1724-38.

69. Balazadeh S, Siddiqui H, Allu AD, Matallana-Ramirez LP, Caldana C, Mehrnia M, Zanor Ml, Köhler B, Mueller-Roeber B. A gene regulatory network controlled by the NAC transcription factor ANAC092/AtNAC2/ORE1 during salt-promoted senescence. Plant J. 2010;62(2):250-64.

70. Xie Q, Frugis G, Colgan D, Chua NH. Arabidopsis NAC1 transduces auxin signal downstream of TIR1 to promote lateral root development. Genes Dev. 2000;14(23):3024-36.

71. Guo HS, Xie Q, Fei JF, Chua NH. MicroRNA directs mRNA cleavage of the transcription factor NAC1 to downregulate auxin signals for Arabidopsis lateral root development. Plant Cell. 2005;17(5):1376-86.

72. Ramamoorthy R, Vishal B, Ramachandran S, Kumar PP. The OsPS1-F gene regulates growth and development in rice by modulating photosynthetic electron transport rate. Plant Cell Rep. 2018;37(2):377-85.

73. E. Montgomery, MB Montgomery. Correlation studies in corn. Agricultural Experiment Station of Nebraska. 1911; pp 108-159.

74. Meyers BC, Axtell MJ, Bartel B, Bartel DP, Baulcombe D, Bowman JL, et al. Criteria for annotation of plant MicroRNAs. Plant Cell. 2008;20(12):3186-90.

75. Addo-Quaye C, Eshoo TW, Bartel DP, Axtell MJ. Endogenous siRNA and miRNA targets identified by sequencing of the Arabidopsis degradome. Curr Biol. 2008;18(10):758-62.

\section{Publisher's Note}

Springer Nature remains neutral with regard to jurisdictional claims in published maps and institutional affiliations.

Ready to submit your research? Choose BMC and benefit from:

- fast, convenient online submission

- thorough peer review by experienced researchers in your field

- rapid publication on acceptance

- support for research data, including large and complex data types

- gold Open Access which fosters wider collaboration and increased citations

- maximum visibility for your research: over $100 \mathrm{M}$ website views per year

At $\mathrm{BMC}$, research is always in progress.

Learn more biomedcentral.com/submissions 